\title{
Vortex Structure in Abelian-Projected Lattice Gauge Theory
}

\author{
J. Ambjørn ${ }^{\mathrm{a}}$, J. Giedt ${ }^{\mathrm{b}}$, J. Greensite ${ }^{\mathrm{c} *}$

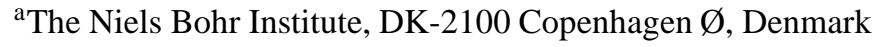 \\ ${ }^{\mathrm{b}}$ Physics Dept., Univ. of California, Berkeley, CA 94720 USA \\ ${ }^{\text {c} P h y s i c s ~ D e p t ., ~ S a n ~ F r a n c i s c o ~ S t a t e ~ U n i v ., ~ S a n ~ F r a n c i s c o, ~ C A ~} 94132$ USA
}

We report on a breakdown of both monopole dominance and positivity in abelian-projected lattice Yang-Mills theory. The breakdown is associated with observables involving two units of the abelian charge. We find that the projected lattice has at most a global $Z_{2}$ symmetry in the confined phase, rather than the global U(1) symmetry that might be expected in a dual superconductor or monopole Coulomb gas picture. Implications for monopole and center vortex theories of confinement are discussed.

Center vortices can be located on thermalized lattices by the technique of center projection in maximal center gauge, and their effects on gaugeinvariant observables such as Wilson loops and topological charge have been clearly seen (e.g. in refs. [1], [2], and in contributions to these Proceedings). A competing theory of confinement is the dualsuperconductor/abelian-projection theory, which has been intensively studied on abelian-projected lattices. It is of some interest to ask if there is evidence of vortex structure also on abelian-projected lattices and, if so, whether this structure is consistent with a picture of the vacuum as a Coulomb gas of monopole loops (for a more detailed presentation of this contribution, cf. [纤).

There is already some evidence that center vortices, in the abelian projection, would appear in the form of a monopole-antimonopole chain, with the $\pm 2 \pi$ monopole flux collimated (at fixed time) in tubelike regions of $\pm \pi$ flux [⿰亻] If If this is so, then several qualitative predictions follow, which can be tested numerically:

- There is $Z_{2}$, rather than $U(1)$, magnetic disorder on finite, abelian-projected lattices;

- Monopole dominance breaks down for even multiples of abelian charge;

- There is strong directionality of field strength

\footnotetext{
${ }^{*}$ Talk presented by J. Greensite. Work supported by the U.S. De-
} partment of Energy under Grant No. DE-FG03-92ER40711. around an abelian monopole, in the direction of the vortex.

Consider large Wilson loops or Polyakov lines on the abelian-projected lattice, corresponding to $q$ units of the abelian electric charge:

$$
\begin{aligned}
W_{q}(C) & =\left\langle\exp \left[i q \oint d x^{\mu} A_{\mu}\right]\right\rangle \\
P_{q} & =\left\langle\exp \left[i q \int d t A_{0}\right]\right\rangle
\end{aligned}
$$

Collimated $\pm \pi$ flux tubes cannot disorder $q=$ even Wilson loops and Polyakov lines. If these vortex tubes are the confining objects, then only for $q=$ odd would we expect $P_{q}=0$, and an area law falloff for Wilson loops. In consequence, there would be $Z_{2}$, rather than $\mathrm{U}(1)$, magnetic disorder/global symmetry in the confined phase.

In contrast, in the monopole Coulomb gas or dual superconductor pictures, we would expect all multiples $q$ of electric charge to be confined; $P_{q}=0$ for all $q$. This is inferred from saddlepoint calculations in $Q E D_{3}$ and strong-coupling calculations in $Q E D_{4}$, it appears to be true for the dual abelian Higgs theory (a model of dual superconductivity), as well as in a simplified treatment of the monopole gas in ref. [5]. The $Z_{2}$ subgroup of $U(1)$ plays no special role in the monopole picture.

Center vortices are rather thick objects $\sim 1 \mathrm{fm}$, so at, e.g., $\beta=2.5$ we would need $12 \times 12 q=2$ Wilson loops to see string breaking. This is impractical. The "fat link" technique is untrustworthy in this case, 


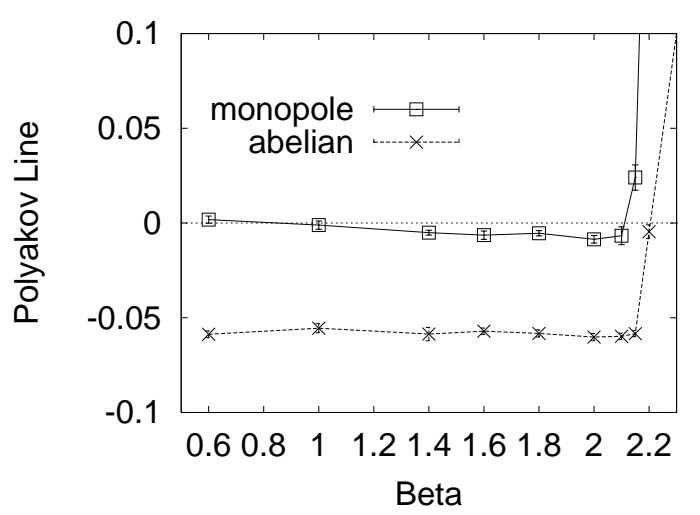

Figure 1. $q=2$ Polyakov lines, $T=3$.

due to the absence of a transfer matrix, and in any case rectangular loops (with $R \gg T$ ) are inadequate; the appropriate operator mixings have to be taken into account. For these reasons, it is best to study $q=$ even Polyakov lines, rather than Wilson loops.

Figure 11 shows our data, in the confined phase, for $q=2$ abelian Polyakov lines on a $12^{3} \times 3$ lattice. The upper line is the monopole dominance (MD) approximation for this quantity, following the method of [6]. We see that $P_{2}$ is finite, negative, and that there is a severe breakdown of the MD approximation in this case. The negative sign is allowed by the absence of reflection positivity in maximal abelian gauge. The finiteness of $P_{2}$ is expected in the center vortex picture, and implies $Z_{2}$ rather than $\mathrm{U}(1)$ disorder on the abelian lattice, while the breakdown of the MD approximation indicates that the abelian monopole flux is not distributed Coulombically.

It is possible to avoid positivity problems by fixing to a spacelike maximal abelian gauge [7]

$R=\sum_{x} \sum_{k=1}^{3} \operatorname{Tr}\left[\sigma_{3} U_{k}(x) \sigma_{3} U_{k}^{\dagger}(x)\right]$ is maximized

What happens in this case is that the loss of positivity is replaced by a breaking of $90^{\circ}$ rotation symmetry. Spacelike $P_{2}$ lines remain negative. Timelike $P_{2}$ lines become positive, although much smaller in magnitude, on a hypercubic lattice, than the spacelike lines. Since this is a physical gauge, the result means that $q=2$ electric charge is unconfined.

It is also interesting to write the link phase angles $\theta_{\mu}(x)$ of the abelian link variables as a sum of the link phase angles $\theta_{\mu}^{M}(x)$ in the MD approximation, plus a so-called "photon" contribution $\theta_{\mu}^{p h}(x) \equiv$ $\theta_{\mu}(x)-\theta_{\mu}^{M}(x)$. It is known that the photon field has no confinement properties at all [6]; Polyakov lines constructed from links $U_{\mu}=\exp \left[i \theta_{\mu}^{p h}\right]$ are finite (also at higher $q$ ), and corresponding Wilson loops have no string tension. Since $\theta_{\mu}^{M}$ would appear to carry all the confining properties, a natural conclusion is that the abelian lattice is indeed a monopole Coulomb gas.

To see that this conclusion may be mistaken, suppose we $a d d$, rather than subtract, the MD angles to the abelian angles, i.e.

$\theta_{\mu}^{\prime}(x)=\theta_{\mu}(x)+\theta_{\mu}^{M}(x)=\theta_{\mu}^{p h}(x)+2 \theta_{\mu}^{M}(x)$

in effect doubling the strength of the monopole Coulomb field. In the monopole picture, this doubling would be expected to increase the $q=1$ string tension, with $P_{1}$ remaining zero. Surprisingly, the opposite occurs; we in fact find that $P_{1}$ is negative in the $\theta^{\prime}$ configurations, with values shown in Table 1 . What

Table 1

\begin{tabular}{|r|r|r|}
\hline \hline $\mathrm{T}$ & $\beta$ & $P_{1}$ line \\
\hline 3 & 1.8 & $-0.0299(20)$ \\
3 & 2.1 & $-0.0405(10)$ \\
4 & 2.1 & $-0.0134(10)$ \\
\hline
\end{tabular}

$q=1$ Polyakov lines on the $\theta^{\prime}$ lattice.

this indicates is that the "photon" and MD contributions do not factorize in Polyakov lines and Wilson loops, contrary to the case in the Villain model. In fact, there is an important and non-perturbative correlation between Polyakov line phases $\theta^{p h}$ and $\theta^{M}$, with the former breaking the (near) U(1) symmetry of the $M D$ lattice down to an exact $Z_{2}$ symmetry. For example, if one computes the average value of $\theta^{p h}$ for $\theta^{M}$ in the intervals $\left[0, \frac{\pi}{2}\right]$ and $\left[\frac{\pi}{2}, \pi\right](\beta=2.1, T=3)$, one finds

$\bar{\theta}^{p h}=\left\{\begin{aligned} 0.027(4) & \text { for } \theta^{M} \in\left[0, \frac{\pi}{2}\right] \\ -0.027(4) & \text { for } \theta^{M} \in\left[\frac{\pi}{2}, \pi\right]\end{aligned}\right.$

The question, of course, is what is the origin of this correlation. From the standpoint of the vortex theory, what is happening is that the Coulombic distribution of $2 \pi$ monopole flux in the MD approximation is 
modified, by its correlation with $\theta^{p h}$, into a configuration with an exact $Z_{2}$ remnant symmetry; confining flux has the same magnitude on the abelian projected and MD lattices, but is distributed differently (collimated vs. Coulombic) at large scales. The negative value of $P_{1}$ in the additive $\theta^{\prime}$ configurations can actually be deduced from the negative value of $P_{2}$ on the abelian projected lattice. For this, we refer the interested reader to ref. [3].

Finally, one would like to see the collimation of field strength, in the neighborhood of an abelian monopole, more directly. Here we have extended the original efforts in ref. [\$] in two ways: First, in the indirect maximal center gauge, we have verified that there is an almost exact alternation of monopoles with antimonopoles along P-vortex lines, as previously conjectured. In the few exceptional cases, there is a static monopole or antimonopole within one lattice spacing of the P-vortex which, if counted as lying along the $\mathrm{P}$-vortex, would restore the exact alternation. Secondly, we have considered spacelike cubes $N=1-4$ lattice spacings wide, pierced on two faces by a single P-vortex, and containing either one or zero static abelian monopoles. We define $W_{n}^{M}(N, N)$ as the vev of unprojected Wilson loops, bounding faces of an $N \times N$ cube containing one static monopole. The subscript $n=0,1$ indicates that the face is pierced $(n=1)$ or unpierced $(n=0)$ by a $\mathrm{P}$ vortex line. $W_{n}^{0}(N, N)$ is the corresponding data for spacelike cubes containing no monopole currents. We then define the fractional deviations

$A_{0,1}^{M}=\frac{W_{0}^{0}(N, N)-W_{0,1}^{M}(N, N)}{W_{0}^{0}(N, N)}$

$A_{0,1}^{0}=\frac{W_{0}^{0}(N, N)-W_{0,1}^{0}(N, N)}{W_{0}^{0}(N, N)}$

The result for 4-cubes is shown in Fig. 目. It is clear that the flux is correlated very strongly with the Pvortex direction, and only rather weakly with the presence or absence of a monopole inside the cube. This is what is expected in the center vortex picture.

We conclude that the (i) non-confinement of $q=$ even abelian electric charge; (ii) breakdown of the monopole dominance approximation; and (iii) highly asymmetric distribution of confining fields around monoples, is consistent with vortex structure on the abelian lattice, but is probably not compatible with

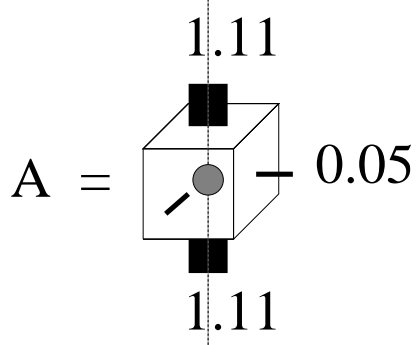

One Monopole

One Vortex

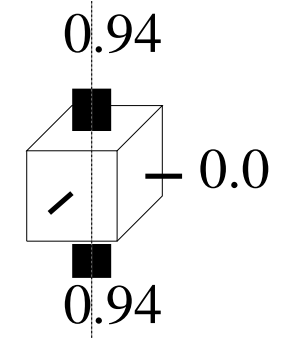

No Monopoles One Vortex

\section{4-Cubes}

Figure 2. Field asymmetry around one and zero monopole 4-cubes. The dashed line indicates cube faces pierced by a P-vortex.

monopole Coulomb gas or dual superconductor pictures. An important point is that charged fields (e.g. off-diagonal gluons) in a confining theory, even if very massive, can have a profound effect on infrared structure. We think it likely that the monopole Coulomb gas and dual-superconductor pictures also break down in the $\mathrm{D}=3$ Georgi Glashow and the Seiberg-Witten models, respectively (cf. the discussion in refs. [3],8]), albeit on a $q=2$ string-breaking scale which increases with the mass of the W-bosons.

\section{REFERENCES}

1. L. Del Debbio, M. Faber, J. Giedt, J. Greensite, Š. Olejník, Phys. Rev. D58(1998) 094501, heplat/9801027.

2. Ph. de Forcrand and M. D'Elia, Phys. Rev. Lett. 82 (1999) 4582, hep-lat/9901020.

3. J. Ambjørn, J. Giedt, J. Greensite, heplat/9907021.

4. L. Del Debbio, M. Faber, J. Greensite, Š. Olejník, Zakopane proceedings, hep-lat/9708023.

5. A. Hart and M. Teper, hep-lat/9902031.

6. T. Suzuki et al., Phys. Lett. B347 (1995) 375, heplat/9408003.

7. M. Chernodub, M. Polikarpov, A. Veselov, JETP Lett. 69 (1999) 174, hep-lat/9812012.

8. J. Ambjørn and J. Greensite, JHEP (1998) 9805:004, hep-lat/9804022. 